\title{
Genetic analysis of superovulatory response of Holstein cows in Canada
}

\author{
C. Jaton, ${ }^{*}{ }^{1}$ A. Koeck, ${ }^{*}$ M. Sargolzaei, ${ }^{*} \ddagger$ F. Malchiodi, ${ }^{*}$ C. A. Price, $\S$ F. S. Schenkel, ${ }^{*}$ and F. Miglior ${ }^{*} \#$ \\ ${ }^{*}$ Centre for Genetic Improvement of Livestock (CGIL), University of Guelph, Guelph, Ontario, Canada, N1G 2W1 \\ †Centre d'insémination artificielle du Québec (Ciaq), Saint-Hyacinthe, Québec, Canada, J2S 7B8 \\ ¥Semex Alliance, Guelph, Ontario, Canada, N1G 3 Z2 \\ §Université de Montréal, Faculté de médecine vétérinaire, St-Hyacinthe, Québec, Canada, J2S 2M2 \\ \#Canadian Dairy Network (CDN), Guelph, Ontario, Canada, N1K 1E5
}

\begin{abstract}
Superovulation of dairy cattle is frequently used in Canada. The cost of this protocol is high, and so is the variability of the outcome. Knowing the superovulatory potential of a donor cow could influence the breeder's decision to superovulate it or not. The main objective of this study was to perform a genetic analysis for superovulatory response of Holstein cows in Canada using data recorded by Holstein Canada, and to investigate if these data could be used for genetic evaluation. Data contained the total number of embryos and the number of viable embryos from every successful flushing performed across Canada. After editing, 137,446 records of superovulation performed between 1992 and 2014 were analyzed. A univariate repeatability animal model analysis was performed for both total number of embryos and number of viable embryos. Because both data and residuals did not follow a normal distribution, records were subject to either logarithmic or Anscombe transformation. Using logarithmic transformation, heritability estimates (SE) of $0.15(0.01)$ and $0.14(0.01)$ were found for total number of embryos and number of viable embryos, respectively. Using Anscombe transformation, heritability estimates (SE) of $0.17(0.01)$ and 0.14 (0.01) were found for total number of embryos and number of viable embryos, respectively. The genetic correlation between the 2 traits was estimated at 0.97 using logarithmic transformation and 0.95 using Anscombe transformation. Breeding values were estimated for 54,463 cows, and 3,513 sires. Only estimated breeding values of sires having a reliability higher than $40 \%$ were considered for estimated breeding values correlations with other routinely evaluated traits. The results showed that selection for a higher response to superovulation would lead to a slight decrease in milk production, but an improvement for functional
\end{abstract}

Received September 3, 2015.

Accepted January 12, 2016.

${ }^{1}$ Corresponding author: cjaton@uoguelph.ca traits, including all reproduction traits. In all cases, the estimated correlations are either low or modest. We conclude that genetic selection for increased superovulatory response in donors is possible; daughters of sires with high estimated breeding values for superovulatory response will tend to yield more embryos, whereas the additive effect of service sire seems not to contribute to the variability of the 2 superovulation traits and was not significantly correlated with the additive effect of the donor.

Key words: superovulation, embryo production, Holstein, genetic parameter, breeding value

\section{INTRODUCTION}

In the 1930s, AI (Hamilton and Symington, 1939) revolutionized the way breeding was done in the dairy industry. Genetically superior bulls were then able to have more offspring, therefore increasing the genetic gain more rapidly throughout the population. Similarly, superovulation and embryo transfer have increased the rate of genetic gain from genetically important cows. This technique has been commercially available since the 1970s as reported by several authors (Farin et al., 2007; Machaty et al., 2012; Hasler, 2014) and is commonly used by dairy producers worldwide. Globally, 700,000 bovine embryos were produced in vivo in 2012, and of that number 350,000 were produced by dairy donors. Also, 440,000 in vitro embryos were produced in the same year (International Embryo Transfer Society, 2012). Superovulation and embryo transfer is used frequently in the Canadian dairy population, with almost 10,000 donors flushed, and over 68,000 transferable embryos collected in 2013 (Canadian Embryo Transfer Association, 2013). In 2012, Canada produced more than $20 \%$ of the global in vivo production of dairy embryos (International Embryo Transfer Society, 2012).

The main disadvantages of superovulation/embryo transfer include cost and the high degree of variability in the number of embryos produced by individual cows (Hahn, 1992; Kanitz et al., 2002; Lonergan and 
Table 1. Summary statistics of the superovulatory data

\begin{tabular}{lrllll}
\hline Item & \multicolumn{1}{c}{ Total } & Average $^{1}$ & SD $^{1}$ & Minimum $^{1}$ & Maximum $^{1}$ \\
\hline No. of records (flush) & 137,446 & - & - & - & - \\
No. of cows & 54,463 & - & - & - & - \\
No. of sires & 3,513 & - & - & - & - \\
No. of service sires & 2,250 & - & - & - & - \\
No. of clinics & 100 & - & - & - & 87 \\
Total no. of embryos & $1,265,333$ & 9.21 & 7.24 & 1 & 58 \\
No. of viable embryos & $1,044,416$ & 7.60 & 5.92 & 0 & \\
\hline${ }^{1}$ On a per flush basis. & & & &
\end{tabular}

Boland, 2011). To date, no genetic analyses have been performed for superovulatory response of dairy cattle in Canada, and no breeding values are available for Canadian producers to select cows based on their superovulatory potential.

Therefore, the main objective of this study was to perform a genetic analysis for superovulatory response of Holstein cows in Canada using data recorded by Holstein Canada, and to investigate if these data could be used for genetic evaluation. The specific objectives were (1) to characterize the superovulatory response data set, (2) to estimate genetic parameters of superovulatory response traits, (3) to estimate breeding values (EBV) for superovulatory response traits and examine their relationship with other routinely evaluated traits in Canada, and (4) to assess the usefulness of having such EBV for the Canadian dairy industry.

\section{MATERIALS AND METHODS}

\section{Data}

Database. Superovulatory response data from April 1980 to March 2014 were obtained from Holstein Canada (Brantford, ON, Canada). Initially, the database consisted of 168,855 records, where one record corresponded to one flush. All records were from Holstein animals. The data set contained information about the donor (identification and birth date), service sire (identification and birth date), date of insemination of the donor, date of embryo recovery, service type, clinic, total number of embryos produced per flush, and number of viable embryos produced per flush. In Canada, all superovulatory protocols are prescribed by veterinarians and the embryos are recovered by the same practitioner. The procedure is usually performed in the barn of the donor's owner. Therefore, in this data set, the clinic corresponds to a veterinarian or group of veterinarians that were involved with the superovulation and embryo transfer of a particular donor.

Data Editing. It is worth noting that only successful flushes with at least one recovered embryo were available, because unsuccessful flushes are not recorded in Canada. Considering the low number of flushes done between 1980 and 1991, only data with a recovery date between 1992 and 2014 were considered in the analysis. In addition, only animals also present in the Canadian Dairy Network database were included in the analysis. If the service sire, birth date, or clinic was missing for a record, it was excluded. Only 3 service types were considered: $\mathrm{H}$, insemination done by a herd owner; $\mathrm{I}$, in vitro; and $\mathrm{T}$, service done by an AI technician. Therefore, any record having another code for service type was deleted. Records were included if the age of the female at recovery was at least 8 mo and no more than 180 mo (15 yr). Duplicate records with exactly the same recovery date, donor, and service sire were deleted. If for one flush, with a given recovery or service date, 2 records were present because 2 different service sires had been used, both records were deleted. Only clinics with 50 or more records were considered. Additionally, 4 clinics had recording problems between 1999 and 2001. Therefore, all the records from those clinics were deleted for those years, as well as records of January 2002 for one of those 4 clinics. Descriptive statistics of the edited data are presented in Table 1.

Trait Definition. The total number of embryos (NE) corresponds to all the embryos recovered from a flush, including degenerated or dead embryos. On the other hand, the number of viable embryos (VE) corresponds to the number of recovered embryos reported to Holstein Canada as transplanted or frozen by the practitioners.

Data Transformation. Test for normality was performed on the raw data and on the residuals of the model used in the analyses (described later) using SAS proc univariate (SAS Institute Inc., 2013). Because both data and residuals for total number of embryos and number of viable embryos did not follow a normal distribution, various transformations were tested to normalize the data. The ones that performed the best were the logarithmic (log) transformation and Anscombe (ans) transformation (Anscombe, 1948). Logarithmic transformation is used to normalize data 
that follows a logarithmic distribution, whereas the Anscombe transformation is used to normalize data that follows a Poisson distribution. Records were therefore transformed as follows:

total number of embryos:

$$
\begin{aligned}
& \mathrm{NE}_{\log }=\ln (\mathrm{NE}), \\
& \mathrm{NE}_{\text {ans }}=2 \times \sqrt{\left(N E+\left(\frac{3}{8}\right)\right)} \\
& \text { number of viable embryos: } \\
& \mathrm{VE}_{\log }=\ln (\mathrm{VE}+1), \\
& \mathrm{VE}_{\text {ans }}=2 \times \sqrt{\left(V E+\left(\frac{3}{8}\right)\right)},
\end{aligned}
$$

Pedigree. An animal pedigree file containing 197,246 animals was generated by tracing the pedigrees of donor cows and service sires with records 7 generations back.

\section{Models}

A univariate and a bivariate repeatability animal model were used to analyze the data, using the average information-REML procedure in the derivative-free approach to multivariate analysis in the DMU package (Madsen and Jensen, 2008).

Univariate Linear Model. Univariate analyses were run for both superovulatory response traits.

To estimate (co-)variances, the following model was run:

$$
\mathbf{y}=\mathbf{X} \boldsymbol{\beta}+\mathbf{Z}_{\mathrm{d}} \mathbf{d}+\mathbf{Z}_{\mathrm{ss}} \mathrm{ss}+\mathbf{Z}_{\mathrm{pe}} \mathbf{p e}+\mathbf{e}
$$

where $\mathbf{y}$ is a vector of logarithmic or Anscombe transformed observations for either total number of embryos or number of viable embryos; $\boldsymbol{\beta}$ is a vector of systematic effects, including fixed effects of age (as linear and quadratic covariate) nested within service type, yearmonth of recovery, clinic-year of recovery, and service type; $\mathbf{d}$ is a vector of random animal additive genetic effects of the donor cow; ss is a vector of random animal additive genetic effects of the service sire; pe is a vector of random permanent environmental effects of the donor; $\mathbf{e}$ is a vector of random residuals; and $\mathbf{X}, \mathbf{Z}_{\mathrm{d}}$, $\mathbf{Z}_{\mathrm{ss}}$, and $\mathbf{Z}_{\mathrm{pe}}$ are the corresponding incidence matrices.

Random effects were assumed to be normally distributed with means equals to zero and covariance structure equal to

$$
\operatorname{Var}\left[\begin{array}{c}
d \\
s s \\
p e \\
e
\end{array}\right]=\left[\begin{array}{cccc}
\mathbf{A} \sigma_{d}^{2} & \mathbf{A} \sigma_{d, s s} & 0 & 0 \\
\mathbf{A} \sigma_{d, s s} & \mathbf{A} \sigma_{s s}^{2} & 0 & 0 \\
0 & 0 & \mathbf{I} \sigma_{p e}^{2} & 0 \\
0 & 0 & 0 & \mathbf{I} \sigma_{e}^{2}
\end{array}\right]
$$

where $\sigma_{d}^{2}, \sigma_{s s}^{2}, \sigma_{p e}^{2}$, and $\sigma_{e}^{2}$ are donor, service sire, permanent environmental, and residual variances, respectively, and $\sigma_{d, s s}$ is the covariance between donor and service sire additive genetic effects. A and $\mathbf{I}$ are additive relationship and identity matrices, respectively.

Because the current version of DMU package does not provide statistical test for fixed effects, the systematic fixed effects included in the previously described mixed model were previously tested for significance using SAS proc HPMIXED (SAS Institute Inc., 2013) accounting for repeated records, and all effects were found highly significant $(P<0.0001)$ by F-test.

Bivariate Linear Model. To estimate the genetic correlation between the 2 superovulatory response traits, a bivariate analysis was run. Fixed and random effects were as in the previously described model. Random effects were assumed to be normally distributed with means equal to zero and covariance structure equal to

$$
\operatorname{Var}\left[\begin{array}{c}
d \\
s s \\
p e \\
e
\end{array}\right]=\left[\begin{array}{cccc}
\mathbf{G}_{0} \otimes \mathbf{A} & \mathbf{G}_{0} \otimes \mathbf{A} & 0 & 0 \\
\mathbf{G}_{0} \otimes \mathbf{A} & \mathbf{G}_{0} \otimes \mathbf{A} & 0 & 0 \\
0 & 0 & \mathbf{P E}_{0} \otimes \mathbf{I} & 0 \\
0 & 0 & 0 & \mathbf{R}_{0} \otimes \mathbf{I}
\end{array}\right],
$$

where $\mathbf{G}_{\mathbf{0}}$ is the additive genetic (co)variance $(2 \times 2)$ matrix between traits; $\mathbf{P E}_{\mathbf{0}}$ is the $(\mathrm{co})$ variance $(2 \times 2)$ matrix between traits due to permanent environmental effects; $\mathbf{R}_{\mathbf{0}}$ is the residual (co)variance $(2 \times 2)$ matrix between traits; and $\otimes$ is the Kronecker product.

\section{Genetic Parameters}

Heritability $\left(\mathrm{h}^{2}\right)$ and repeatability $(\mathrm{r})$, were calculated as follows. For the donor $(d)$ :

$$
\begin{gathered}
h_{d}^{2}=\sigma_{d}^{2} /\left(\sigma_{d}^{2}+2 \sigma_{d, s s}+\sigma_{s s}^{2}+\sigma_{p e}^{2}+\sigma_{e}^{2}\right), \\
r_{d}=\left(\sigma_{d}^{2}+\sigma_{p e}^{2}\right) /\left(\sigma_{d}^{2}+2 \sigma_{d, s s}+\sigma_{s s}^{2}+\sigma_{p e}^{2}+\sigma_{e}^{2}\right),
\end{gathered}
$$

and for the service sire $(s s)$ :

$$
h_{s s}^{2}=\sigma_{s s}^{2} /\left(\sigma_{d}^{2}+2 \sigma_{d, s s}+\sigma_{s s}^{2}+\sigma_{p e}^{2}+\sigma_{e}^{2}\right) .
$$




\section{Breeding Value Estimation}

Breeding values of donors and their sires for total number of embryos and number of viable embryos were estimated from univariate analyses. Reliabilities of $\operatorname{EBV}\left(r_{i}^{2}\right)$ were calculated as follows:

$$
r_{i}^{2}=1-\frac{P E V_{i}}{\hat{\sigma}_{d}^{2}},
$$

where $\mathrm{PEV}_{\mathrm{i}}$ is the predicted error variance of animal $i$, and $\hat{\sigma}_{d}^{2}$ is the estimated additive genetic variance of the donor effect used to estimate the trait EBV.

Pearson correlations between EBV of sires with a minimum reliability of $40 \%$ and official genomic breeding values for routinely evaluated traits were computed for total number of embryos and number of viable embryos.

\section{RESULTS AND DISCUSSION}

\section{Descriptive Statistics}

The number of flushes per year has increased since 1992 and seems to have reached a plateau of about 8,000 flushes per year (Figure 1). Fifty-three percent of the donors included in the data set were flushed once, and $90 \%$ were flushed 5 times or less (Figure 2).

Mean values (SD) for total number of embryos and number of viable embryos per flush were 9.2 (7.2) and 7.6 (5.9), respectively. Those values are higher than those reported by other studies (Table 2) because this

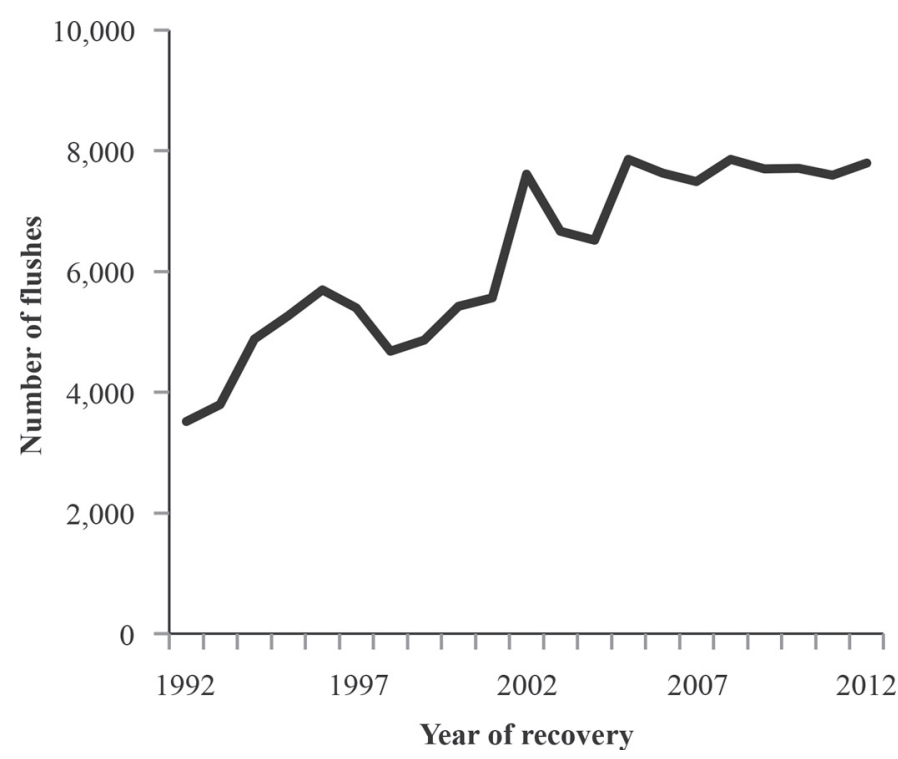

Figure 1. Number of flushes per year of recovery after data editing. data set does not include unsuccessful flushes. The distribution of the number of embryos per flush is presented in Figure 3.

Recovery Year. Figure 4 shows the average number of embryos per flush by year of recovery. For both traits, a similar increasing trend is observed over the years. Because there has been no genetic selection for superovulatory response in Canada, this increase could be attributed to the improvement of the technical aspects of superovulation.

Recovery Month. Over the summer months (June to September), a very small decrease seems to be present for the superovulatory response of dairy cattle (Figure 5), likely due to the heat stress that cattle may undergo during summer. Many authors have reported a reduction in embryo quality (Sartori et al., 2002; Chebel et al., 2008; Vieira et al., 2014) and response to superovulation (Hasler et al., 1983; Lonergan and Boland, 2011) in animals exposed to heat stress.

Clinic. Figure 6 shows the average values for the total number of embryos and the number of viable embryos per flush for the 100 clinics included in the analysis, where each dot represents a clinic. As shown in this figure, a considerable difference was found between the best and worst clinics that performed superovulation and embryo recovery. The best clinic recovers on average 9.5 embryos and 5.3 viable embryos per flush more than the worst clinic.

Service Type. The average number of embryos per flush per service type is presented in Figure 7. Overall, the number of embryos recovered after superovulation is slightly higher when the service is performed by an AI technician, and lower when the protocol is performed in vitro. Considering the higher cost of producing embryos in vitro, usually only very valuable cows with reproductive problems or very valuable young heifers undergo an in vitro protocol (Hasler, 2003; Mapletoft and Hasler, 2005). This may partly explain why fewer embryos were produced in vitro.

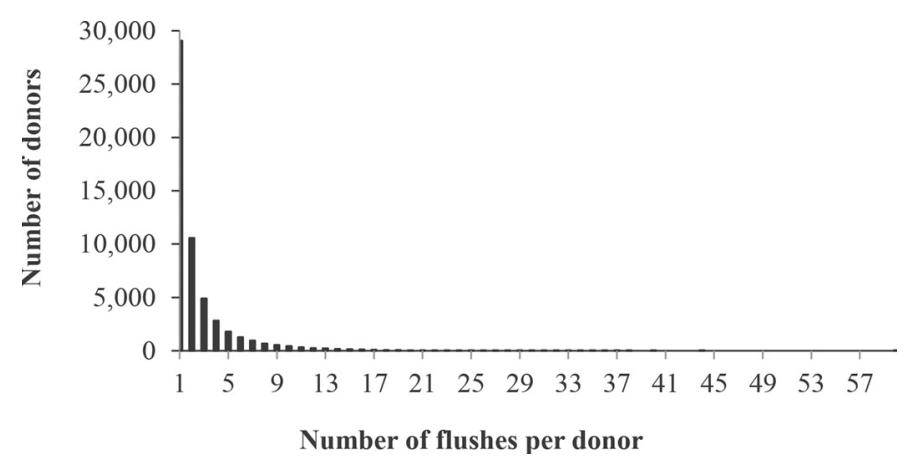

Figure 2. Number of flushes per donor. 

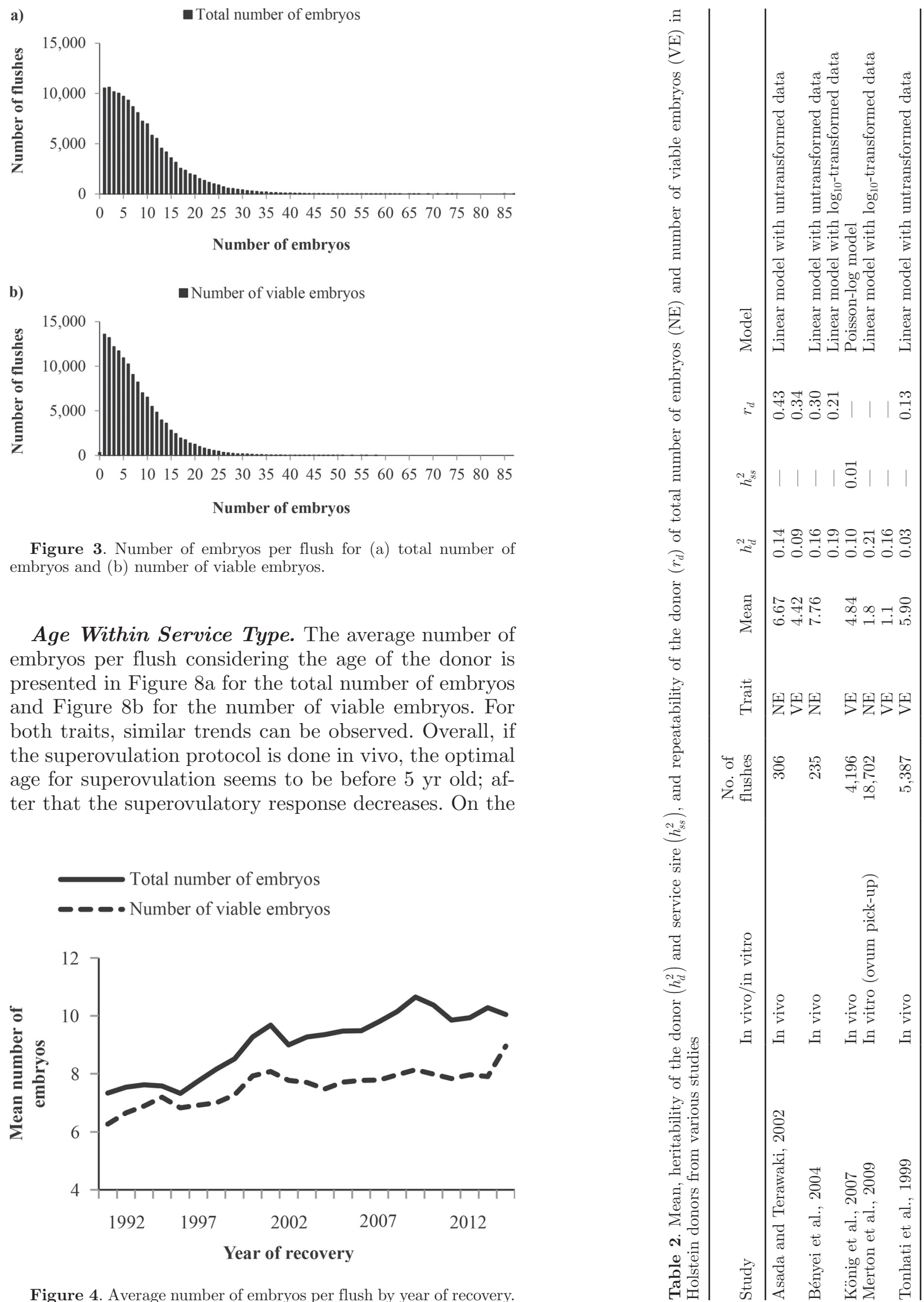

Figure 3. Number of embryos per flush for (a) total number of embryos and (b) number of viable embryos.

Age Within Service Type. The average number of embryos per flush considering the age of the donor is presented in Figure 8a for the total number of embryos and Figure $8 \mathrm{~b}$ for the number of viable embryos. For both traits, similar trends can be observed. Overall, if the superovulation protocol is done in vivo, the optimal age for superovulation seems to be before $5 \mathrm{yr}$ old; after that the superovulatory response decreases. On the

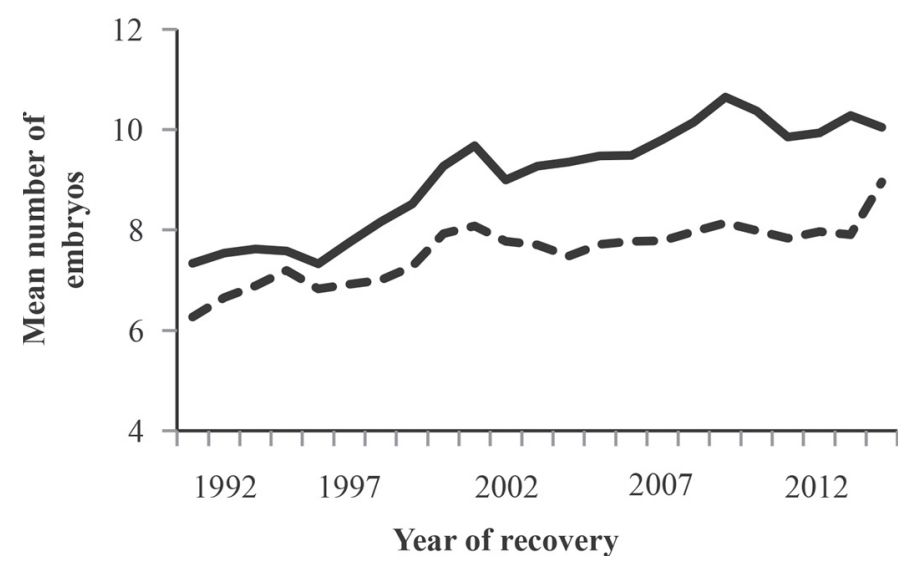

Figure 4. Average number of embryos per flush by year of recovery. 


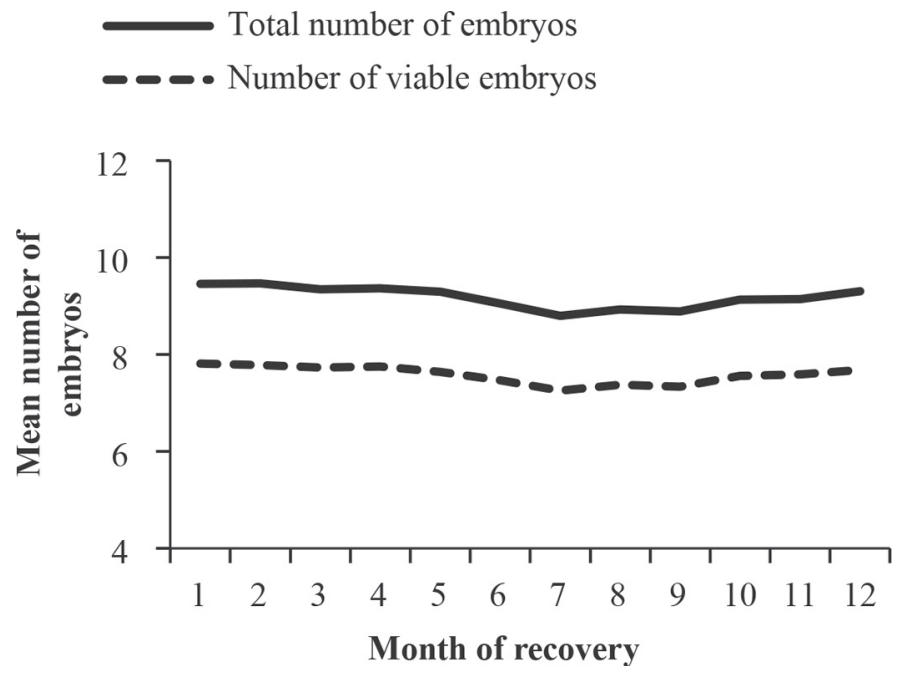

Figure 5. Average number of embryos per flush by month of recovery.

other hand, protocols performed in vitro yield more constant results no matter the age of the donor. Some studies did not find a significant difference between the embryo production of donors of different ages (Kafi and McGowan, 1997; Hasler, 2014; Vieira et al., 2014). One study reported that heifers produced less embryos then mature cows, and that at $10 \mathrm{yr}$ old the superovulatory response starts to decline (Hasler et al., 1983). Another study indicated that third lactation cows produced more oocytes after being superovulated than first lactation cows, but no comparisons with older cows were performed (Snijders et al., 2000).

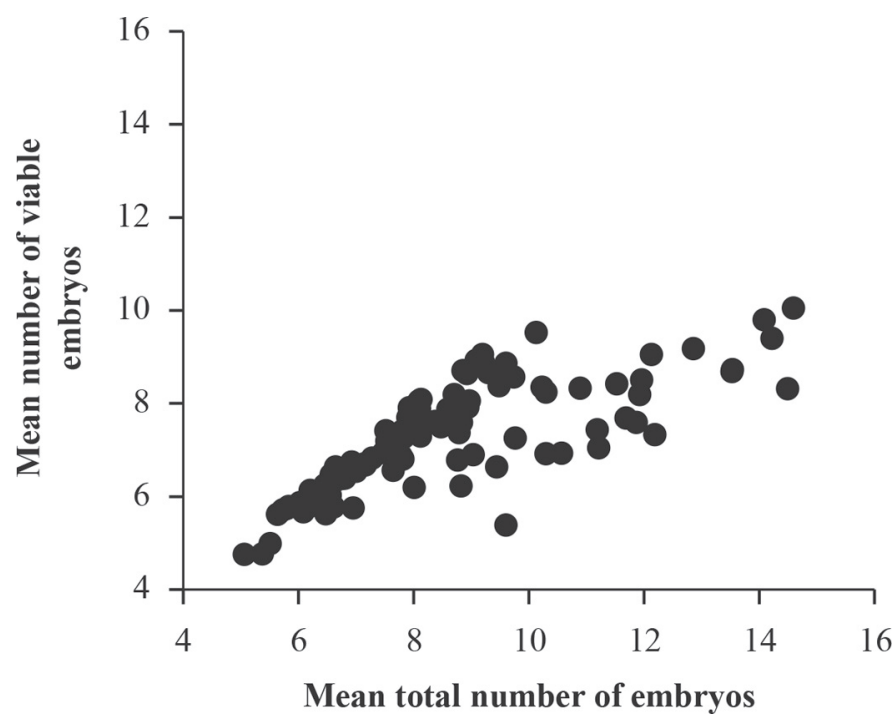

Figure 6. Average number of embryos per flush by clinic (each dot represents a single clinic).
Total number of embryos

Number of viable embryos

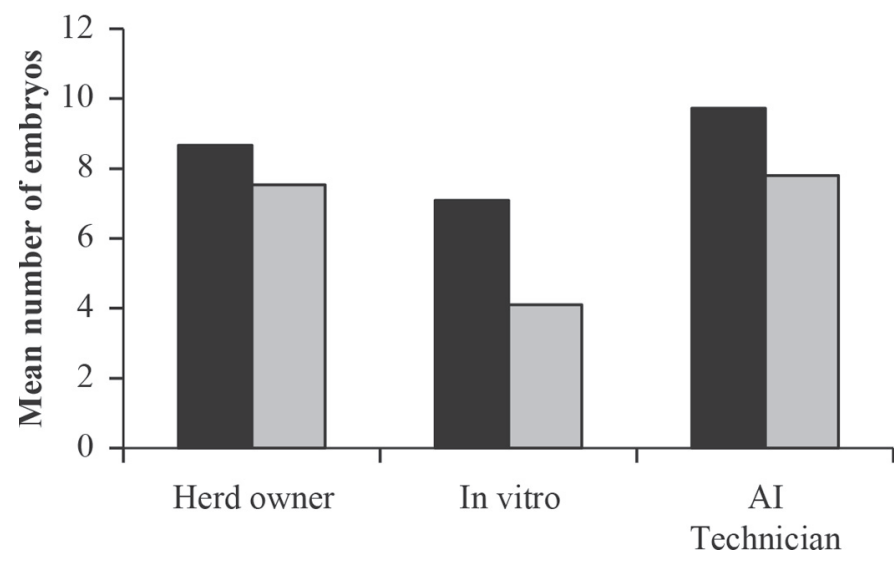

Figure 7. Average number of embryos per flush by service type.

\section{Genetic Parameters}

Heritability. Heritability estimate from the univariate model for the donor effect, using log-transformed data, for total number of embryos was $0.15 \pm 0.01$, which is slightly higher than the $0.14 \pm 0.01$ heritability estimated for the number of viable embryos. Using the Anscombe transformation slightly higher heritabilities were found: $0.17 \pm 0.01$ for total number of embryos and $0.14 \pm 0.01$ for number of viable embryos (Table 3). Only few other studies have estimated heritability with regard to superovulatory response of Holstein donors in a large data set. Table 2 summarized the results of similar studies done with Holstein cattle. Heritability estimates ranged from 0.03 to 0.21 , showing that environmental factors strongly affect superovulation of dairy cattle (Bényei et al., 2004), but it is a heritable trait (Mapletoft, 2006; Yang et al., 2010; Cory et al., 2013). Overall, the results obtained in this study were in the same range of previous estimates.

Heritability estimates for the service sire were very low for both superovulatory response traits: $0.007 \pm$ 0.001 (log-transformation) and $0.006 \pm 0.001$ (Anscombe transformation) for the total number of embryos and $0.014 \pm 0.002$ ( $\log$ and Anscombe transformation) for the number of viable embryos (Table 3). This is in accordance with the heritability of the service sire for the number of viable embryos of 0.01 found by König et al. (2007).

Repeatability. Repeatability was 0.24 and 0.23 for total number of embryos and number of viable embryos, respectively, using the log-transformed data. Using the Anscombe transformation, repeatabilities of 0.28 and 0.24 were obtained for total number of embryos and number of viable embryos, respectively (Table 3 ). 
a)

Herd owner $\quad \square$ AI technician $\quad \square$ in vitro

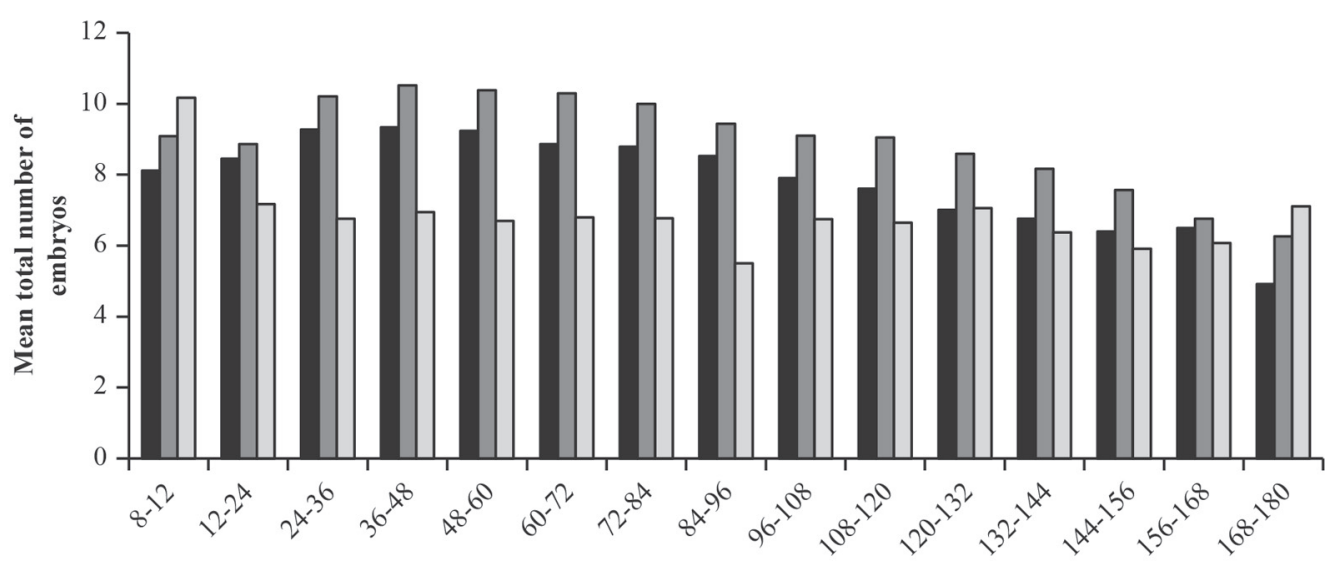

b)

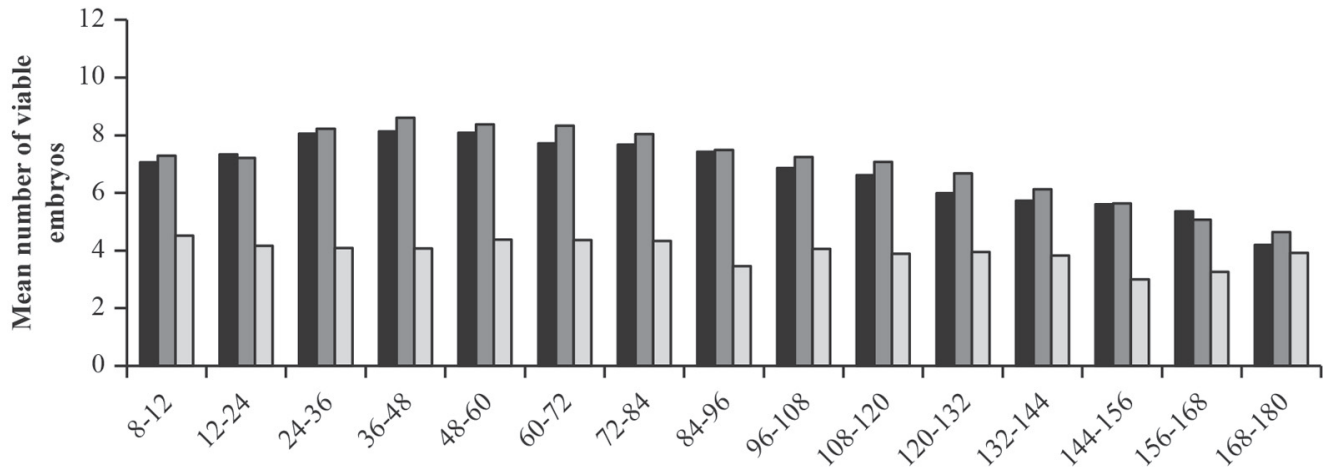

Age of the donor (mo)

Figure 8. Average number of embryos per flush by age within service type for (a) total number of embryos and (b) number of viable embryos.

These values are in the same range as reported in the literature (Table 2). As mentioned previously, superovulatory response has a large variability, but many authors agree that the number of embryos produced by a superovulated female is repeatable within an animal (Lohuis, 1995; Asada and Terawaki, 2002; Mapletoft, 2006; Lonergan and Boland, 2011). Moreover, it was reported that daughters of cows that respond well to

Table 3. Genetic parameter estimates from univariate analyses for the total number of embryos (NE) and the number of viable embryos (VE) using logarithm (Log) or Anscombe (Ans) transformation of the data (SE in parentheses; SE was not available for repeatability of the donor)

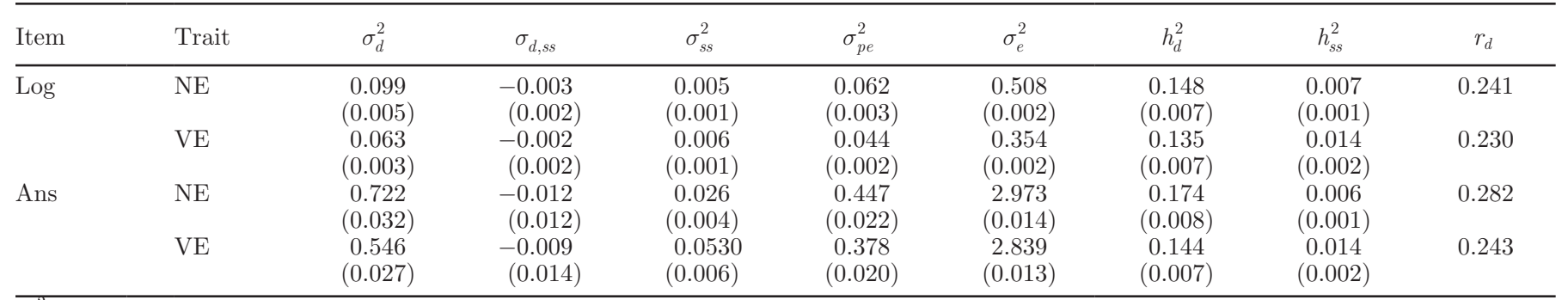

$\overline{1 \sigma_{d}^{2},} \sigma_{s s}^{2}, \sigma_{p e}^{2}$, and $\sigma_{e}^{2}$ are donor, service sire, permanent environmental and residual variances, respectively, and $\sigma_{d, s s}$ is the covariance between donor and service sire additive genetic effects, $h_{d}^{2}$ is the heritability of the donor, $h_{s s}^{2}$ is the heritability of the service sire, and $r_{d}$ is the repeatability of the donor. 
Table 4. Estimates of genetic parameters from the bivariate analysis for total number of embryos (NE) and number of viable embryos (VE) using log-transformed data ${ }^{1}$

\begin{tabular}{|c|c|c|c|c|}
\hline Item & NE_donor & NE_service sire & VE_donor & VE_service sire \\
\hline NE_donor & $\begin{array}{r}\mathbf{0 . 1 4 8} \\
(0.007)\end{array}$ & $\begin{array}{c}-0.085 \\
(0.085)\end{array}$ & $\begin{array}{c}0.969 \\
(0.004)\end{array}$ & $\begin{array}{c}-0.005 \\
(0.080)\end{array}$ \\
\hline NE_service sire & & $\begin{array}{r}\mathbf{0 . 0 0 8} \\
(0.001)\end{array}$ & $\begin{array}{c}-0.105 \\
(0.087)\end{array}$ & $\begin{array}{c}0.976 \\
(0.007)\end{array}$ \\
\hline VE_donor & & & $\begin{array}{r}\mathbf{0 . 1 3 4} \\
(0.007)\end{array}$ & $\begin{array}{c}-0.036 \\
(0.082)\end{array}$ \\
\hline VE_service sire & & & & $\begin{array}{r}\mathbf{0 . 0 1 5} \\
(0.002)\end{array}$ \\
\hline
\end{tabular}

${ }^{1}$ Heritability in bold on the diagonal and genetic correlation between traits above the diagonal with $\mathrm{SE}$ in parentheses.

superovulation are likely to also have a good response (Mapletoft, 2006).

Genetic Correlations. The genetic correlation between the service sire and the donor effects was near zero and negative for both traits using both transformations (Tables 4 and 5). Those correlations are in agreement with the results from König et al. (2007), who applied a similar model. This implies that selection for increased superovulatory response from the donor will have no effect on the fertility of the service sires.

Genetic correlations of 0.97 (log transformation) and 0.95 (Anscombe transformation) were estimated between the total number of embryos and the number of viable embryos. High correlations were expected because the 2 traits are highly phenotypically correlated (log-transformed data: $\mathrm{r}_{\mathrm{p}}=0.89$; Anscombetransformed data: $\left.\mathrm{r}_{\mathrm{p}}=0.88\right)$.

\section{Breeding Value Estimation}

Because EBV from the log-transformed and Anscombe-transformed data were highly correlated (Pearson correlation of 0.98), only results from the log-transformed data are presented hereafter. Similarly, considering that the EBV from univariate and bivariate analysis were highly correlated for total numbers of embryos (Pearson correlation of 0.99) and number of viable embryos (Pearson correlation of 0.95 ), the breeding values from the univariate analysis were used.

EBV Correlations With Other Routinely Evaluated Traits. The EBV correlations of superovulatory response traits with other routinely evaluated traits in Canada were calculated using the EBV of sires that had reliability equal or higher than $40 \%$ (Table 6 ). It should be noted that, with the exception of SCS, higher EBV scores are desirable.

Both total number of embryos and number of viable embryos had similar correlations with each of the traits. The correlation of EBV for superovulatory traits with EBV for production traits such as milk, fat, and protein yield was significant, although in all cases, it was modest, but negative, implying that high-producing cows tend to have a reduced response to superovulation. König et al. (2007) found similar results, obtaining a genetic correlation of -0.27 for milk yield and the number of viable embryos. High-producing dairy cows have been reported to have decreased fertility (Pryce et al., 2004), suggesting a possible effect of milk yield on reproduction traits. However, a study showed that milk production did not have an effect on the number of embryos produced by a superovulated donor, but had an effect on the fertilization rate (Hasler et al., 1983).

Table 5. Estimates of genetic parameters from the bivariate analysis for total number of embryos (NE) and number of viable embryos (VE) using Anscombe-transformed data ${ }^{1}$

\begin{tabular}{|c|c|c|c|c|}
\hline Item & NE_donor & NE_service sire & VE_donor & VE_service sire \\
\hline NE_donor & $\begin{array}{r}\mathbf{0 . 1 7 5} \\
(0.008)\end{array}$ & $\begin{array}{c}-0.053 \\
(0.084)\end{array}$ & $\begin{array}{c}0.953 \\
(0.005)\end{array}$ & $\begin{array}{c}0.005 \\
(0.077)\end{array}$ \\
\hline NE_service sire & & $\begin{array}{c}\mathbf{0 . 0 0 7} \\
(0.001)\end{array}$ & $\begin{array}{c}-0.076 \\
(0.087)\end{array}$ & $\begin{array}{c}0.976 \\
(0.008)\end{array}$ \\
\hline VE_donor & & & $\begin{array}{c}\mathbf{0 . 1 4 0} \\
(0.007)\end{array}$ & $\begin{array}{c}-0.033 \\
(0.080)\end{array}$ \\
\hline VE_service sire & & & & $\begin{array}{r}\mathbf{0 . 0 1 5} \\
(0.002)\end{array}$ \\
\hline
\end{tabular}

${ }^{1}$ Heritability in bold on the diagonal and genetic correlation between traits above the diagonal with SE in parentheses. 
Another study on in vitro produced embryos also demonstrated that the number of oocytes and the quality of the oocytes recovered from high- or low-producing dairy cows were not significantly different (Snijders et al., 2000).

The small negative correlation between superovulatory traits and the SCS, and its small positive correlation with mastitis resistance (Table 6), is in accordance with the data of König et al. (2007). This implies that improving udder health through lower SCS or higher mastitis resistance selection will also tend to have a favorable effect on the number of embryos produced.

Functional traits, such as direct herd life and BCS, had a positive correlation with the response to superovulation, which is in accordance with the literature. Authors have argued that the donor should preferably be gaining weight (Farin et al., 2007) and have no nutritional deficiencies (Mapletoft, 2006; Velazquez, 2011), as undernutrition is deleterious to follicular development and embryo quality (Stroud and Hasler, 2006; Lonergan and Boland, 2011). It is also important that the animal is not overfed because its superovulatory response could be reduced (Stroud and Hasler, 2006; Velazquez, 2011). Moreover, a study demonstrated that heifers with too high BCS $(>3.50)$ tended to give a lower number of embryos, but more research needs to be done to confirm those findings (Kadokawa et al., 2008).

Reproduction traits of heifers and cows were also favorably correlated with superovulatory response. This was expected because superovulation is also a reproduction trait. Some authors recommend making sure that the donor has no reproductive problems before starting superovulation (Mapletoft, 2006; Farin et al., 2007) because cows that have reproductive problems are usually poor donors (Hasler et al., 1983; Farin et al., 2007; Lonergan and Boland, 2011).

Finally, the modest negative correlation between LPI, which is the Canadian national selection index, and superovulatory response traits indicates that the current global selection in Canada will not increase the number of embryos produced by Holstein cattle.

For all routinely evaluated traits considered, however, the estimated correlations were either low or modest, making superovulatory response a new trait that would convey unique information compared with what is already recorded in the Canadian dairy industry.

Best and Worst Sires and Donors. Sire EBV from univariate analyses with a reliability of at least $40 \%$ in the data set were compared with the average phenotype of their daughters. Comparison between the best (10\% quantile) and worst (90\% quantile) sires according to their EBV for superovulatory traits is shown in Table 7. Daughters of the sires with the best EBV had on average 10.97 embryos and 9.18 viable embryos, whereas daughters of sires with the worst EBV had on average 7.05 embryos and 5.57 viable embryos.

A similar analysis was performed using the donor EBV with a reliability of at least $40 \%$. The average phenotype of the best (10\% quantile) and worst (90\%

Table 6. Pearson correlations between EBV of sires from univariate analyses with a minimum reliability of $\geq 40 \%$ for total number of embryos and number of viable embryos and EBV of routinely evaluated traits $(\mathrm{n}=$ number of sires)

\begin{tabular}{llcc}
\hline \multirow{2}{*}{ Item } & Trait & $\begin{array}{c}\text { Total no. of } \\
\text { embryos } \\
(\mathrm{n}=1,391)\end{array}$ & $\begin{array}{c}\text { No. of viable } \\
\text { embryos } \\
(\mathrm{n}=1,251)\end{array}$ \\
\hline \multirow{3}{*}{ Production } & LPI ${ }^{1}$ & $-0.23^{* * *}$ & $-0.14^{* * *}$ \\
& Milk yield & $-0.26^{* * *}$ & $-0.21^{* * *}$ \\
Udder health & Protein yield & $-0.29^{* * *}$ & $-0.24^{* * *}$ \\
& Fat yield & $-0.21^{* * *}$ & $-0.15^{* * *}$ \\
Reproduction & SCS & $-0.08^{* *}$ & $-0.12^{* *}$ \\
& Mastitis resistance & $0.10^{* *}$ & $0.10^{*}$ \\
& Daughter fertility & $0.20^{* * *}$ & $0.20^{* * *}$ \\
& 56-d nonreturn rate in heifers & $0.16^{* * *}$ & $0.16^{* * *}$ \\
& 56-d nonreturn rate in cows & $0.15^{* * *}$ & $0.14^{* * *}$ \\
& No. of services in heifers & $0.20^{* * *}$ & $0.21^{* * *}$ \\
& No. of services in cows & $0.21^{* * *}$ & $0.21^{* * *}$ \\
Longevity & First service to conception in heifers & $0.19^{* * *}$ & $0.20^{* * *}$ \\
& First service to conception in cows & $0.21^{* * *}$ & $0.22^{* * *}$ \\
& Calving to first service & $0.12^{* * *}$ & $0.12^{* *}$ \\
& Days open & $0.21^{* * *}$ & $0.21^{* * *}$ \\
& Herd life & $0.07^{*}$ & $0.12^{* * *}$ \\
& Direct herd life & $0.07^{* *}$ & $0.12^{* * *}$ \\
& BCS & $0.25^{* * *}$ & $0.20^{* * *}$ \\
\hline
\end{tabular}

${ }^{1}$ Canadian national selection index.

${ }^{*} P<0.05,{ }^{* *} P<0.01,{ }^{* * *} P<0.001$. 
Table 7. Average number of embryos of best (10\% quantile) and worst (90\% quantile) sires, with a reliability of $\mathrm{EBV}$ of $\geq 40 \%$, according to their EBV for total number of embryos (NE) and number of viable embryos (VE) from a univariate linear animal model

\begin{tabular}{lcccc} 
& & \multicolumn{3}{c}{ Mean number of embryos of the daughters } \\
\cline { 3 - 5 } Trait & No. of & Mean & $10 \%$ quantile & $90 \%$ quantile \\
\hline NE & sires & 9.21 & 10.97 & 7.05 \\
VE & 1,391 & 7.53 & 9.18 & 5.57 \\
\hline
\end{tabular}

quantile) donors according to their EBV were compared (Table 8). There was a larger difference of 11.34 embryos and 9.91 viable embryos between the best and worst donors.

\section{Limitations}

A limitation of this study is that unsuccessful flushes are currently not recorded in Canada, and it would be interesting to have this information in the future. Having information about the unsuccessful flushes would give results that represent the reality even more. However, even without this information, the results presented in this study are similar to the ones from other studies that had information about unsuccessful flushes, maybe because of the very large size of this data set. The results presented in this study therefore provide interesting information for the dairy industry for successful superovulatory response of donors.

Another limitation of this study is that no information was available about the herd where the superovulatory protocol was performed. Donors are elite cows that often change owner, which makes it hard to know their herd when the superovulation protocol was performed. The herd effect should be important to consider in future work because it accounts for feeding and management of the donors. However, usually only a few donors are superovulated in a herd. The limited number of observations per herd could make this effect hard to model.

A final limitation of this study may be that the donor cows with records in the database were elite cows that were selected for their high genetic evaluations or high phenotypic performances. Therefore, they may not rep- resent the Canadian Holstein population entirely, which may lead to some sort of selection bias in the results. However, it is important to notice that the superovulated donors were not selected considering their superovulatory potential, but rather on other traits, which are not genetically correlated or are weakly genetically correlated with superovulatory potential, minimizing the possible selection bias. In addition, estimated genetic trends for the 2 superovulatory traits show a flat trend overtime (i.e., no genetic change), reflecting the absence of genetic selection for these traits in the database.

\section{Overall Remarks}

The results of the present study suggest that genetic selection for increased superovulatory response in donors is possible. Daughters of sires with high EBV for superovulatory response will tend to yield more embryos; however, the additive effect of service sire seems not to contribute significantly to the variability of the 2 superovulation traits.

Having genetic information about superovulatory response could be useful for dairy producers that flush elite females. They could choose females that are more likely to yield a larger number of viable embryos based on their EBV for superovulatory response. Moreover, producers that often buy animals in sales are usually interested to flush females to more rapidly improve their herds. It would be interesting for them to know the superovulatory potential of a female before the purchase.

Considering that this new breeding value might not be of interest for all dairy producers, it might not be

Table 8. Average number of embryos of best (10\% quantile) and worst (90\% quantile) donors, with a reliability of EBV of $\geq 40 \%$, according to their EBV for total number of embryos (NE) and number of viable embryos (VE) from a univariate linear animal model

\begin{tabular}{lcccc}
\hline & & \multicolumn{3}{c}{ Mean number of embryos } \\
\cline { 3 - 5 } Trait & No. of & Mean & $10 \%$ quantile & $90 \%$ quantile \\
\hline NE & 32,403 & 9.23 & 15.61 & 4.27 \\
VE & 29,554 & 7.62 & 13.40 & 3.49 \\
\hline
\end{tabular}


important to include it in a national selection index such as LPI, but it still could be available through the Canadian Dairy Network for producers that are interested in producing embryos. Even if superovulatory traits are not yet included in breeding goals, it may be of interest to genetically select for these traits to improve the efficiency of embryo production (Merton et al., 2009).

Having breeding values for superovulatory response could also be of interest for the AI industry and for veterinarians or researchers. For the AI industry, it could help promote some bulls with a high EBV for superovulatory response, therefore increasing the chances that their daughters will produce a higher number of embryos. Veterinarians or researchers might also have interest in knowing the superovulatory potential of a donor to adjust the superovulatory protocol accordingly and to optimize the number of embryos produced by a donor. More research would be needed to find different superovulatory protocols that could be used according to the superovulatory potential of a donor.

\section{CONCLUSIONS}

Selection for higher superovulatory response in Holstein cows in Canada is possible and could be an additional tool available to Canadian dairy breeders. It could mainly be used to identify outliers before making a decision to superovulate a donor or not. Correlations between breeding values of superovulatory traits with other routinely recorded traits showed that selection for a higher response to superovulation would lead to a slight decrease in milk, fat, and protein yields, but an improvement for functional traits, including all reproduction traits. However, in all cases, the estimated correlations were either low or moderate, indicating that selection for cows with high production performance that produce large number of viable embryos is possible.

\section{ACKNOWLEDGMENTS}

The authors are thankful to Holstein Canada for its contribution in providing the data. This study was funded by Ciaq (St-Hyacinthe, Québec, Canada), Semex (Guelph, Ontario, Canada), the DairyGen Council of the Canadian Dairy Network (Guelph, Ontario, Canada), and the Natural Sciences and Engineering Research Council of Canada (Ottawa, Ontario, Canada).

\section{REFERENCES}

Anscombe, F. J. 1948. The transformation of Poisson, binomial and negative-binomial data. Biometrika 35:246-254.
Asada, Y., and Y. Terawaki. 2002. Heritability and repeatability of superovulatory responses in Holstein population in Hokkaido, Japan. Asian-australas. J. Anim. Sci. 15:944-948.

Bényei, B., A. Gáspárdy, I. Komlósi, and A. Pécsi. 2004. Repeatability and heritability of ovulation number and embryos in damdaughters pairs in superovulated Holstein-Friesian cows. Reprod. Domest. Anim. 39:99-102.

Canadian Embryo Transfer Association. 2013. Summary of embryo transfer activity in Canada for 2013. Accessed Mar. 5, 2015. http://www.ceta.ca/pdfs/2014/2013-ET-Activity-in-Canada.pdf.

Chebel, R. C., D. G. B. Demétrio, and J. Metzger. 2008. Factors affecting success of embryo collection and transfer in large dairy herds. Theriogenology 69:98-106.

Cory, A. T., C. A. Price, R. Lefebvre, and M. F. Palin. 2013. Identification of single nucleotide polymorphisms in the bovine folliclestimulating hormone receptor and effects of genotypes on superovulatory response traits. Anim. Genet. 44:197-201.

Farin, P. W., K. Moore, and M. Drost. 2007. Assisted reproductive technologies in cattle. Pages 496-508 in Large Animal Theriogenology. Saunders Elsevier, St. Louis, MO.

Hahn, J. 1992. Attempts to explain and reduce variability of superovulation. Theriogenology 38:269-275.

Hamilton, J. G., and E. L. Symington. 1939. Cooperative artificial insemination of dairy cattle. Can. J. Comp. Med. 3:337-340.

Hasler, J. F. 2003. The current status and future of commercial embryo transfer in cattle. Anim. Reprod. Sci. 79:245-264.

Hasler, J. F. 2014. Forty years of embryo transfer in cattle: A review focusing on the journal Theriogenology, the growth of the industry in North America, and personal reminisces. Theriogenology $81: 152-169$

Hasler, J. F., A. D. McCauley, E. C. Schermerhorn, and R. H. Foote. 1983. Superovulatory responses of Holstein cows. Theriogenology 19:83-99.

International Embryo Transfer Society. 2012. 2012 statistics of embryo collection and transfer in domestic farm animals. Accessed Mar. 5, 2015. http://www.iets.org/pdf/comm_data/december2013.pdf.

Kadokawa, H., N. Tameoka, M. Uchiza, Y. Kimura, and M. Yonai. 2008. Short communication: A field study on the relationship between body condition and embryo production in superovulated Holstein yearling heifers. J. Dairy Sci. 91:1087-1091.

Kafi, M., and M. R. McGowan. 1997. Factors associated with variation in the superovulatory response of cattle. Anim. Reprod. Sci. 48:137-157.

Kanitz, W., F. Becker, F. Schneider, E. Kanitz, C. Leiding, H. P. Nohner, and R. Pöhland. 2002. Superovulation in cattle: Practical aspects of gonadotropin treatment and insemination. Reprod. Nutr. Dev. 42:587-599.

König, S., F. Bosselmann, U. U. von Borstel, and H. Simianer. 2007. Genetic analysis of traits affecting the success of embryo transfer in dairy cattle. J. Dairy Sci. 90:3945-3954.

Lohuis, M. M. 1995. Potential benefits of bovine embryo-manipulation technologies to genetic improvement programs. Theriognology 43:51-60.

Lonergan, P., and M. P. Boland. 2011. Gamete and embryo technology | Multiple ovulation and embryo transfer. Pages 623-630 in Encyclopedia of Dairy Sciences (Second Edition). J. W. Fuquay, ed. Academic Press, San Diego, CA.

Machaty, Z., J. Peippo, and A. Peter. 2012. Production and manipulation of bovine embryos: Techniques and terminology. Theriogenology 78:937-950.

Madsen, P., and J. Jensen. 2008. An user's guide to DMU. A package for analyzing multivariate mixed models. Version 6 , release 4.7. Danish Institute of Agricultural Sciences, Tjele, Denmark.

Mapletoft, R. J. 2006. Bovine embryo transfer. IVIS Rev. Vet. Med., International Veterinary Information Service, Ithaca, NY.

Mapletoft, R. J., and J. F. Hasler. 2005. Assisted reproductive technologies in cattle: A review. Rev. Sci. Tech. Off. Int. Epizoot. 24:393-403.

Merton, J. S., B. Ask, D. C. Onkundi, E. Mullaart, B. Colenbrander, and M. Nielen. 2009. Genetic parameters for oocyte number and 
embryo production within a bovine ovum pick-up-in vitro production embryo-production program. Theriogenology 72:885-893.

Pryce, J. E., M. D. Royal, P. C. Garnsworthy, and I. L. Mao. 2004. Fertility in the high-producing dairy cow. Livest. Prod. Sci. 86:125-135.

Sartori, R., R. Sartor-Bergfelt, S. A. Mertens, J. N. Guenther, J. J. Parrish, and M. C. Wiltbank. 2002. Fertilization and early embryonic development in heifers and lactating cows in summer and lactating and dry cows in winter. J. Dairy Sci. 85:2803-2812.

SAS Institute Inc. 2013. SAS/STAT ${ }^{\circledR} 13.1$ User's Guide. SAS Institute Inc., Cary, NC.

Snijders, S. E., P. Dillon, D. O'Callaghan, and M. P. Boland. 2000. Effect of genetic merit, milk yield, body condition and lactation number on in vitro oocyte development in dairy cows. Theriogenology 53:981-989.

Stroud, B., and J. F. Hasler. 2006. Dissecting why superovulation and embryo transfer usually work on some farms but not on others. Theriogenology 65:65-76.
Tonhati, H., R. B. Lôbo, and H. N. Oliveira. 1999. Repeatability and heritability of response to superovulation in holstein cows. Theriogenology 51:1151-1156.

Velazquez, M. A. 2011. The role of nutritional supplementation on the outcome of superovulation in cattle. Anim. Reprod. Sci. 126:1-10.

Vieira, L. M., C. A. Rodrigues, M. F. Mendanha, M. F. Sá Filho, J. N. S. Sales, A. H. Souza, J. E. P. Santos, and P. S. Baruselli. 2014. Donor category and seasonal climate associated with embryo production and survival in multiple ovulation and embryo transfer programs in Holstein cattle. Theriogenology 82:204-212.

Yang, W. C., S. J. Li, K. Q. Tang, G. H. Hua, C. Y. Zhang, J. N. Yu, L. Han, and L. G. Yang. 2010. Polymorphisms in the $5^{\prime}$ upstream region of the FSH receptor gene, and their association with superovulation traits in Chinese Holstein cows. Anim. Reprod. Sci. 119:172-177. 